\title{
The effects of organic fertilizer and inorganic fertilizer on yield and
}

\section{quality of lettuce}

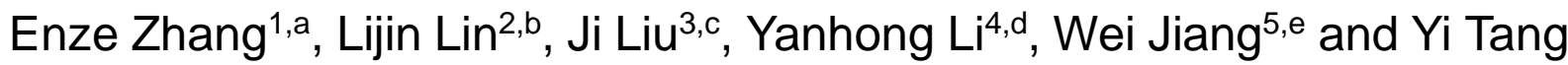 \\ $2, f *$
}

${ }^{1}$ College of Horticulture, Sichuan Agricultural University, Chengdu, Sichuan, China ${ }^{2}$ Institute of Pomology and Olericulture, Sichuan Agricultural University, Chengdu, Sichuan, China

${ }^{3}$ Chengdu Academy of Agriculture and Forestry Sceinces, Chengdu, Sichuan, China

${ }^{4}$ Chengdu Hongke Agricultural Investment Company Limited, Chengdu, Sichuan, China

${ }^{5}$ College of Chemistry and Life Science, Chengdu Normal University, Chengdu, Sichuan, China

a570081509@qq.com, ㄲllj800924@qq.com, c34905418@qq.com, d418961103@qq.com, e1399945180@qq.com, 'tangyisunguochao@sina.com

${ }^{*}$ Corresponding author. Enze Zhang and Lijin Lin contributed equally to this work.

Keywords: lettuce; organic fertilizer; inorganic fertilizer; quality

Abstract: In order to study the organic fertilizer, inorganic fertilizer and its effect on the yield and quality of lettuce, a pot experiment with 5 treatments (no fertilizer, single organic fertilizer and inorganic fertilizer, organic - inorganic fertilizer, organic - inorganic fertilizer (1/2)) was conducted. Treated with organic - inorganic fertilizer, the yield of lettuce increased $60.67 \%, 37.12 \%$ and $14.84 \%$, while compared with treatment of no fertilizer, single manure and inorganic fertilizers. Meanwhile, quality of lettuce improved significantly after application of fertilizer, in which organic - inorganic fertilizer showed the best results.

\section{Introduction}

Vegetables have the nutritional characteristics of shallow root system, the weak ability of root system to absorb nutrients, being fond of fertilizer and so on. It often is met the quality of the fertilizer effect in vegetable fertilization process [1]. Inorganic fertilizer can provide the nutrition that vegetables need. Studies shows lots of inorganic fertilizer can be bad at the vegetables' growth by the comparison of the yield and quality of cucumbers under different fertilizer rates [2]. Li discovers the phenomenon of "high yield and poor quality", that using organic fertilizer, on the basis of inorganic fertilizer, can increase production as well as increase the content of nitrate in lettuce [3].

Lettuce (Lactuca sativa L.) is easy to govern, the economic benefit is higher, and its growth period is short, so the domestic cultivation common around the world [3]. In this study, we use organic fertilizer, inorganic fertilizer and organic-inorganic fertilizer to lettuce and compare the changes in the yield and quality, to found out the best way to use fertilizer for lettuce.

\section{Materials and Methods}

Materials. The experiments were conducted at Sichuan Agricultural University, Wenjiang, China. 
The seeds of lettuce named Italy 338 were purchased from Chengdu, China. Soil pH is 6.67 7.90, organic matter is $18.8 \sim 3.23 \mathrm{~g} \cdot \mathrm{kg}^{-1}$, total nitrogen is $2.30 \sim 4.33 \mathrm{~g} \cdot \mathrm{kg}^{-1}$, total phosphorus is $13.8 \mathrm{mg} \cdot \mathrm{kg}^{-1}$, total potassium $10 \mathrm{mg} \cdot \mathrm{kg}^{-1}$. The fertilizer that were purchased from Chengdu Lihebao biological fertilizer co., LTD is convenient and available potassium sulphate compound fertilizer.

Experimental Design. Fertilizer every processing is added in every pot at once and blends with soil before engraftment. No fertilizer and pesticide is applied during lettuce growth. There are five processing that consist of 6 pots with one plant per pot. Every processing repeats 3 times. The specific fertilization methods in different processing are shown in table 1. 15 lettuces are harvested after the mature. Plant height measured with scale, quality with electronic scales. The soluble sugar content is determined by anthrone colorimetry. Soluble protein content is determined by coonmassie brilliant white G250 method. Vc content is determined by molybdenum blue colorimetry [4]. Chlorophyll content is determined by spectrophotometry [5].

Table 1 Different fertilizer treatment

\begin{tabular}{cccccc}
\hline Fertilizer $\left(\mathrm{g} \cdot\right.$ pot $\left.^{-1}\right)$ & \multicolumn{5}{c}{ Processing } \\
\hline & $\mathrm{CK}$ & $\mathrm{A}$ & $\mathrm{B}$ & $\mathrm{C}$ & $\mathrm{D}$ \\
Organic F & 0 & 48 & 24 & 48 & 0 \\
Inorganic F & 0 & 0.6 & 0.3 & 0 & 0.6 \\
\hline
\end{tabular}

Statistic analyses. Statistical analyses were performed using SPSS 13.0 statistical software (IBM, Chicago, IL, USA). Data were analyzed by one-way ANOVA with least significant difference (LSD) at a $5 \%$ confidence level.

\section{Results and Discussion}

Single-plant Weight (Spw). Using fertilizer enhanced Spw of lettuce significantly, compared with CK (Fig. 1). Compared single organic F (C), single inorganic F (D) enhanced Spw by 19\% $(\mathrm{p}<0.05)$. It was shown that the effect of inorganic F, enhancing Spw, was better than organgic F. The inorganic-organic F processing (A) enhanced Spw best. It enhanced Spw by $37 \%(\mathrm{p}<0.05)$ compared with single organic $F(C)$ and by $15 \%(p<0.05)$ compared with single inorganic $F(D)$. The processing $(\mathrm{A})$ is the most effective to ehance Spw.

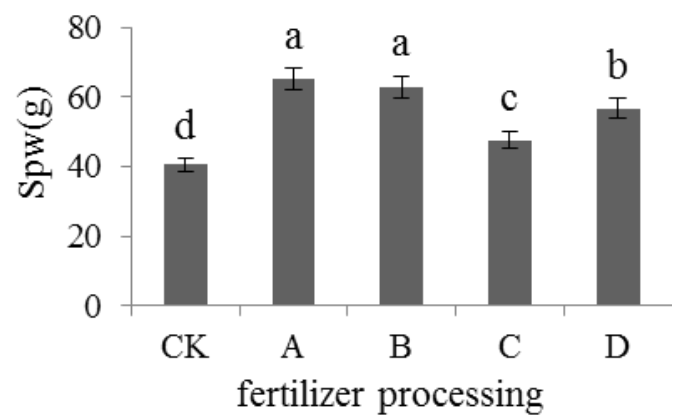

Fig.1. The influence of different fertilizer treatments on Spw

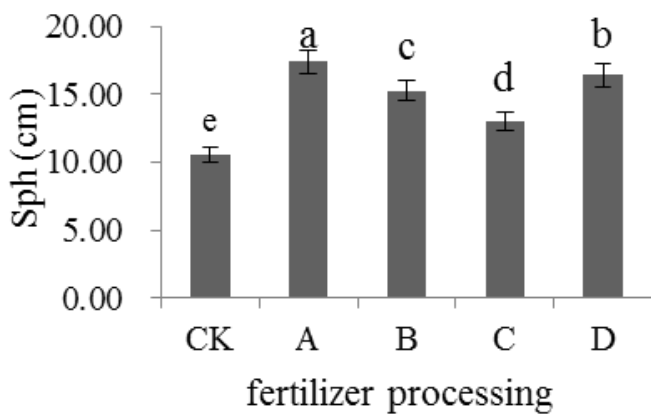

Fig.2. The influence of different fertilizer treatments on Sph

Single-plant Height (Sph). Using fertilizer enhanced Sph of lettuce significantly, compared with $\mathrm{CK}$ (Fig. 2). The height every processing is $\mathrm{A}>\mathrm{D}>\mathrm{B}>\mathrm{C}>\mathrm{CK}(\mathrm{p}<0.05)$. Compared with single 
organic F (C), single inorganic F (D) enhanced Sph by 26\%. Compared with each processing, it was inorganic F why Sph was enhanced. The inorganic-organic F processing (A) enhanced Spw best. It enhanced Spw by 34\% compared with single organic $\mathrm{F}(\mathrm{C})$ and by $6 \%$ compared with single inorganic F (D). The processing (A) is the most effective to ehance Sph.

Vitamin C Content (Vcc). Using fertilizer enhanced Vcc of lettuce significantly, compared with CK (Fig. 3). Compared with single inorganic F (D), single organic F (C) enhanced VCc by $12 \%$ $(\mathrm{p}<0.05)$. It was shown that the organic $\mathrm{F}$ was the important reason why VCc was enhanced. The inorganic-organic F processing (A) enhanced Vcc best. It enhanced Vcc by $9 \%(\mathrm{p}<0.05)$ compared with single organic $F(C)$ and by $22 \%(\mathrm{p}<0.05)$ compared with single inorganic $F(D)$. The processing (A) is the most effective to ehance Vcc.

Soluble Protein Content (Spc). Using fertilizer enhanced Spc of lettuce significantly, compared with CK (Fig. 4). Compared with single inorganic F (D), single organic F (C) enhanced Spc by $1 \%$ $(\mathrm{p}<0.05)$. It was shown that the organic $\mathrm{F}$ was the important reason why Spc was enhanced. The inorganic-organic F processing (A) enhanced Spc best. It enhanced Spc by $12 \%(\mathrm{p}<0.05)$ compared with single organic $F(C)$ and by $20 \%(\mathrm{p}<0.05)$ compared with single inorganic $F(D)$. The processing $(\mathrm{A})$ is the most effective to ehance

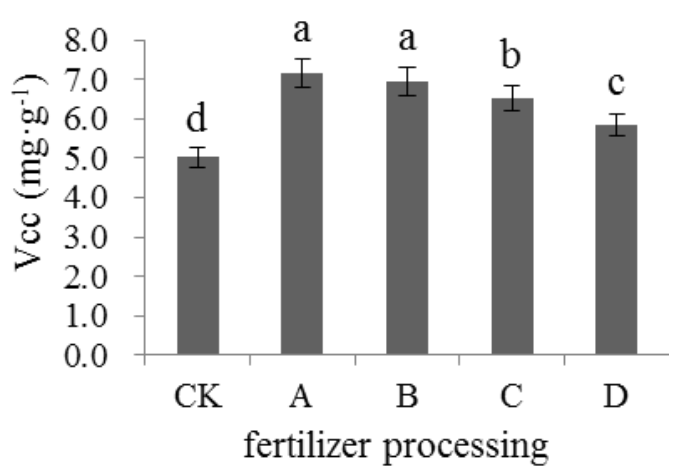

Fig.3. The influence of different fertilizer treatments on Vcc

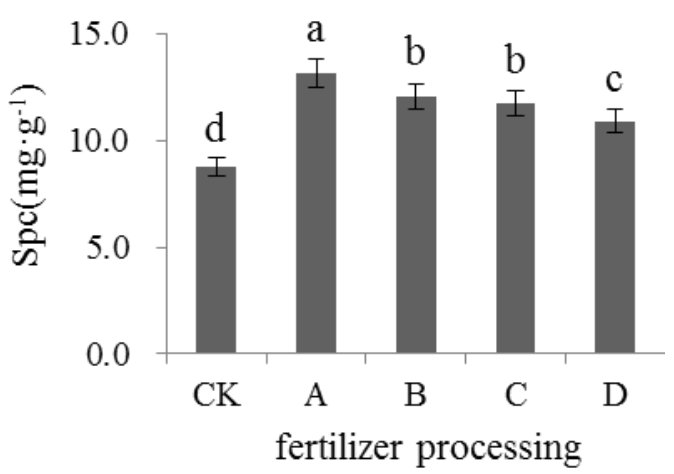

Fig.4. The influence of different fertilizer treatments on $\mathrm{Spc}$

Soluble Sugar Content (Ssc). Using fertilizer enhanced Ssc of lettuce significantly, compared with CK (Fig. 5). Compared with single organic F (C), single inorganic F (D) enhanced Ssc by $21 \%$ $(\mathrm{p}<0.05)$. It was shown that the inorganic $\mathrm{F}$ was the important reason why Ssc was enhanced. The inorganic-organic F processing (A) enhanced Ssc best. It enhanced Ssc by 39\% ( $<<0.05)$ compared with single organic $\mathrm{F}(\mathrm{C})$ and by $15 \%(\mathrm{p}<0.05)$ compared with single inorganic $\mathrm{F}(\mathrm{D})$. The processing (A) is the most effective to ehance Ssc.

Total Chlorophyll Content (Tcc). Using fertilizer enhanced Tcc of lettuce significantly, compared with CK (Fig. 6). Compared with single organic F (C), single inorganic F (D) enhanced Tcc by $12 \%$ $(p<0.05)$. It was shown that the inorganic $F$ was the important reason why Tcc was enhanced. The inorganic-organic F processing (A) enhanced Tcc best. It enhanced Tcc by $39 \%$ ( $\mathrm{p}<0.05$ ) compared with single organic $\mathrm{F}(\mathrm{C})$ and by $15 \%(\mathrm{p}<0.05)$ compared with single inorganic $\mathrm{F}(\mathrm{D})$. The processing $(\mathrm{A})$ is the most effective to ehance Tcc. 


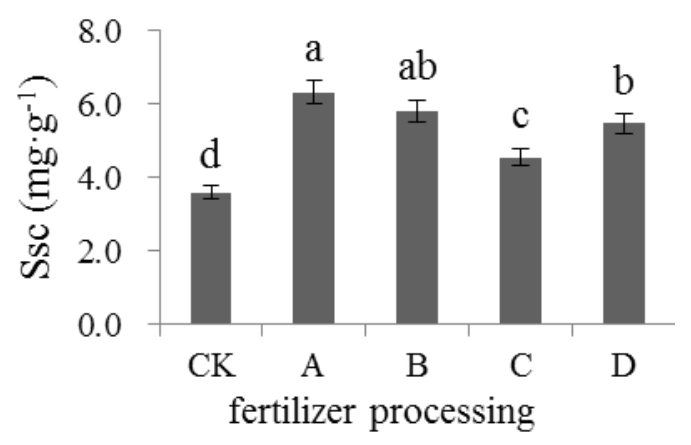

Fig.5. The influence of different fertilizer treatments on Ssc

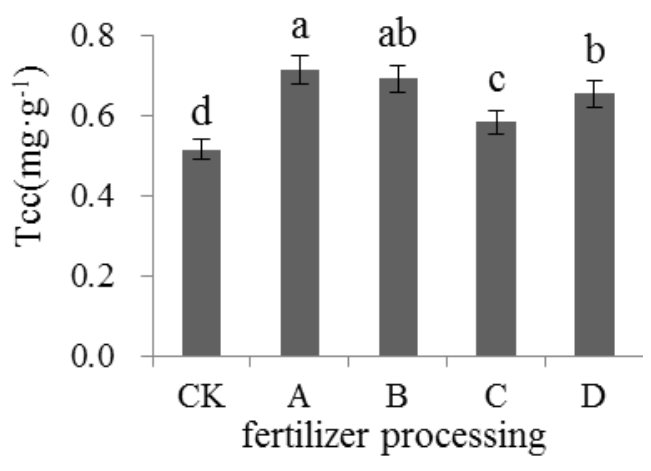

Fig.6. The influence of different fertilizer treatments on Tcc

\section{Conclusions}

The result showed that using fertilizer is the imortant measure to enhance yield and quality of lettuce, but there was difference between different fertilizer treatments. In terms of Spw, Sph,Ssc and Tcc, organic F have greater advantages. In terms of Vcc and Spc, inorganic $\mathrm{F}$ have greater advantages Single organic F and single inorganic F were less effective for lettuce. Compared with them, organic-inorganic F not only play advantages of inorganic $\mathrm{F}$ available and high yied but also can improve Vcc, Spc, Ssc and Tcc effectively [6]. At the same time, it was found that how much organic-inorganic $\mathrm{F}$ under the same fertlization had no obvious effect on the yield and quality of lettuce [7]. The research about using organic-inorganic $\mathrm{F}$ at once was an important measure to improve the yield and quality of lettuce. The way had a certain application value in lettuce production.

\section{References}

[1] Z. Q. Zhang, W. X. Zhao and W. B. Zhang. Transactions of the CSAE Vol. 21(2005), p. 159-161.

[2] J. Guo, Z. L. Li. Beijing Agriculture 27(2013).

[3]H. H. Li, Z. Y. Wang. Journal of Agricultural University of Hebei Vol. 41 (2007), p. 29-32.

[4] H. S. Li. Beijing: HEP, 2000.

[5] X. Y. Wang. China Agricultural Information (First Half ) Vol. 3 (2014), p. 211-212.

[6] L. Q. Zhou, H. W. Tan, M. F. Huang. Guangxi Agricultural Science Vol. 39 (2008), p. 192-195.

[7] H. Y. Xu, R. Q. Li. Modern Agricultural Science and Technology Vol. 15(2009), p. 71-72. 